\title{
La représentation du savoir chez l'enseignant, source de difficultés dans l'enseignement de certaines connaissances
}

\section{Sylvette Maury}

\section{(2) OpenEdition \\ Journals \\ Édition électronique \\ URL : http://journals.openedition.org/trema/2436 \\ DOI : $10.4000 /$ trema.2436 \\ ISSN : 2107-0997 \\ Éditeur \\ Faculté d'Éducation de l'université de Montpellier}

Édition imprimée

Date de publication : 1 mai 1992

Pagination : 75-80

ISSN : 1167-315X

\section{Référence électronique}

Sylvette Maury, «La représentation du savoir chez l'enseignant, source de difficultés dans l'enseignement de certaines connaissances », Tréma [En ligne], 1 | 1992, mis en ligne le 01 mai 1992, consulté le 19 avril 2019. URL : http://journals.openedition.org/trema/2436 ; DOI : 10.4000/ trema.2436

Ce document a été généré automatiquement le 19 avril 2019.

Trema 


\title{
La représentation du savoir chez l'enseignant, source de difficultés dans l'enseignement de certaines connaissances
}

\author{
Sylvette Maury
}

1 Le domaine des probabilités et celui des représentations graphiques ont en commun une importance sociale qui va croissant avec le développement de l'informatique, en grande partie du fait qu'ils interviennent tous deux de manière significative dans le traitement et l'analyse des données statistiques. Or paradoxalement, on observe une grande résistance du système éducatif à l'enseignement effectif de ces secteurs. Ainsi, par exemple, les éléments de statistique ou de probabilités, qui figurent dans les programmes de très nombreuses classes, ont fréquemment fait, dans le passé, l'objet "d'allégements » (officieux ou officiels), allègements qui résultaient généralement d'un accord (tacite ou explicite selon les cas) entre divers partenaires: enseignants, corps d'inspection, associations de spécialistes...

2 A l'heure où les programmes de collège et de lycée prennent en compte de manière significative ces domaines de la connaissance, il est important de s'interroger sur cette «résistance » : elle pourrait, en effet, se manifester malgré une pression sociale et une demande institutionnelle fortes.

3 Les facteurs qui contribuent à freiner le développement de l'enseignement des probabilités et des représentations graphiques sont, sans nul doute, divers. Le but de l'article est de mettre en évidence l'un d'entre eux qui intervient, selon nous, de manière notable dans les deux domaines de la connaissance auxquels nous nous intéressons ici : il s'agit de la représentation qu'ont les enseignants du savoir de référence. Pour fonder ce point de vue, nous allons examiner séparément le domaine des probabilités et celui des représentations graphiques. 


\section{Les probabilités} plutôt de l'ordre épistémologique, alors que d'autres renvoient au niveau conceptuel.

En premier lieu, on trouve chez les enseignants une interrogation sur les fondements des probabilités. Celle-ci n'est pas en général formulée comme telle ; elle apparaît plutôt sous une forme qui semble traduire un souci pédagogique. Il s'agit, pour le professeur, de savoir quel est le type d'approche préférable dans l'enseignement :

- l'approche dite « empirique ", avec laquelle la probabilité est calculée (dans les cas simples) à partir de l'observation de la fréquence relative d'une même issue lors d'épreuves répétées ${ }^{1}$ ;

- l'approche dite «théorique » avec laquelle (dans les mêmes cas simples) la probabilité est obtenue en effectuant le rapport entre les nombres de cas favorables et de cas possibles ${ }^{2}$.

6 Cette interrogation que l'on rencontre aussi chez des probabilistes professionnels ou qui se manifeste dans les brusques changements de programmes ${ }^{3}$ peut traduire, comme nous le soulignions plus haut, un simple souci pédagogique. Cependant, nous pensons que, dans bien des cas, elle dissimule en fait une interrogation sur la légitimité de telle ou telle approche (légitimité par rapport au cadre des mathématiques) ou sur sa validité, c'est-àdire sur sa capacité à représenter un certain aspect de la réalité.

7 Ajoutons que, à un niveau d'enseignement ou de spécialisation plus élevé en probabilités, on n'échappe guère aux interrogations épistémologiques ; celles-ci peuvent se manifester, en particulier, à travers des conflits ou des choix « d'écoles » : statistique classique versus statistique «Bayésienne »; enseignement «classique» des probabilités versus théorie axiomatique des probabilités ${ }^{4}$.

Un deuxième type de difficultés, en probabilités, provient de ce que dans la vie courante, tous les individus dès leur plus jeune âge et hors enseignement, sont constamment confrontés à des phénomènes aléatoires. Il se forme ainsi chez eux (y compris chez ceux qui deviendront ultérieurement professeurs de mathématiques!) des «intuitions » probabilistes, c'est-à-dire en quelque sorte, des modèles qui se développent en dehors de toute intention d'enseignement. Ces modèles ont un domaine de validité parfois assez étendu, en tous les cas non vide, mais évidemment ils ne coïncident pas, en général, avec le modèle mathématique. Que se passe-t-il alors lorsqu'un individu reçoit un enseignement de probabilités? Il se développe chez lui, dans les cas favorables, des conceptions correctes ; mais les intuitions erronées n'en disparaissent pas pour autant et dans certaines situations, même chez les individus ayant un bon niveau de formation en probabilités, les intuitions erronées peuvent se trouver mobilisées. Cela les professeurs de mathématiques en ont l'expérience. Par exemple, s'agissant d'une situation probabiliste, lequel d'entre eux n'a jamais été surpris par l'écart entre le résultat issu d'un calcul théorique et l'attente intuitive qu'il en avait, sur laquelle il aurait été prêt à parier !

Pour nous, ce décalage entre les prévisions associées au modèle mathématique et celles provenant de certaines intuitions erronées, créerait un sentiment d'angoisse, angoisse elle-même amplifiée par l'incertitude relative aux fondements. En définitive, le malaise ainsi engendré contribuerait, au niveau global, à freiner le développement de l'enseignement des probabilités. 


\section{Les représentations graphiques}

10 Avec les représentations graphiques, la situation est sensiblement différente. En effet, si les probabilités, en dépit d'une formation initiale généralement insuffisante des professeurs (car la résistance à l'enseignement de ce secteur est importante également à l'université!), sont perçues comme faisant partie intégrante du savoir mathématique établi, cela n'est pas le cas des représentations graphiques. Pourtant, leur enseignement est essentiellement à la charge des professeurs de mathématiques et cela de manière très nette depuis que les nouveaux programmes des collèges ont été mis en place.

11 En fait, le problème qui semble se poser en premier aux enseignants, à l'abord du champ des représentations graphiques, est celui de l'identification du savoir. En effet :

- d'une part, les professeurs n'ont jamais été familiarisés avec la sémiologie graphique qui peut être considérée comme le savoir de référence (il faut dire que les ouvrages de cette discipline, dans lesquels sont expliquées les techniques d'élaboration des représentations graphiques et leurs conditions d'utilisation, sont très spécialisés et très peu diffusés) ;

- d'autre part, la question du savoir à enseigner, qui n'est pas tout à fait la même, se pose avec acuité ; en effet, traditionnellement, les représentations graphiques ne sont pas perçues comme un objet d'enseignement et il n'y a pas eu, dans ce domaine, capitalisation de connaissances pédagogiques liées à la pratique. Pour illustrer notre propos, nous allons présenter deux exemples d'exercices tels qu'on peut les trouver dans les manuels scolaires des collèges.

Dans le premier exemple, les populations de trois villes A, B, C sont communiquées aux élèves (habitants : hts).

A : 50000 hts B : 80000 hts C : 90000 hts

Dans le deuxième exemple ce sont les populations d'une même ville à trois dates différentes qui leur sont communiquées :

1950 : 50000 hts $1970: 80000$ hts $1980: 90000$ hts

Les graphiques traditionnellement associés à ces deux situations sont représentés ciaprès. 
Exemple 1

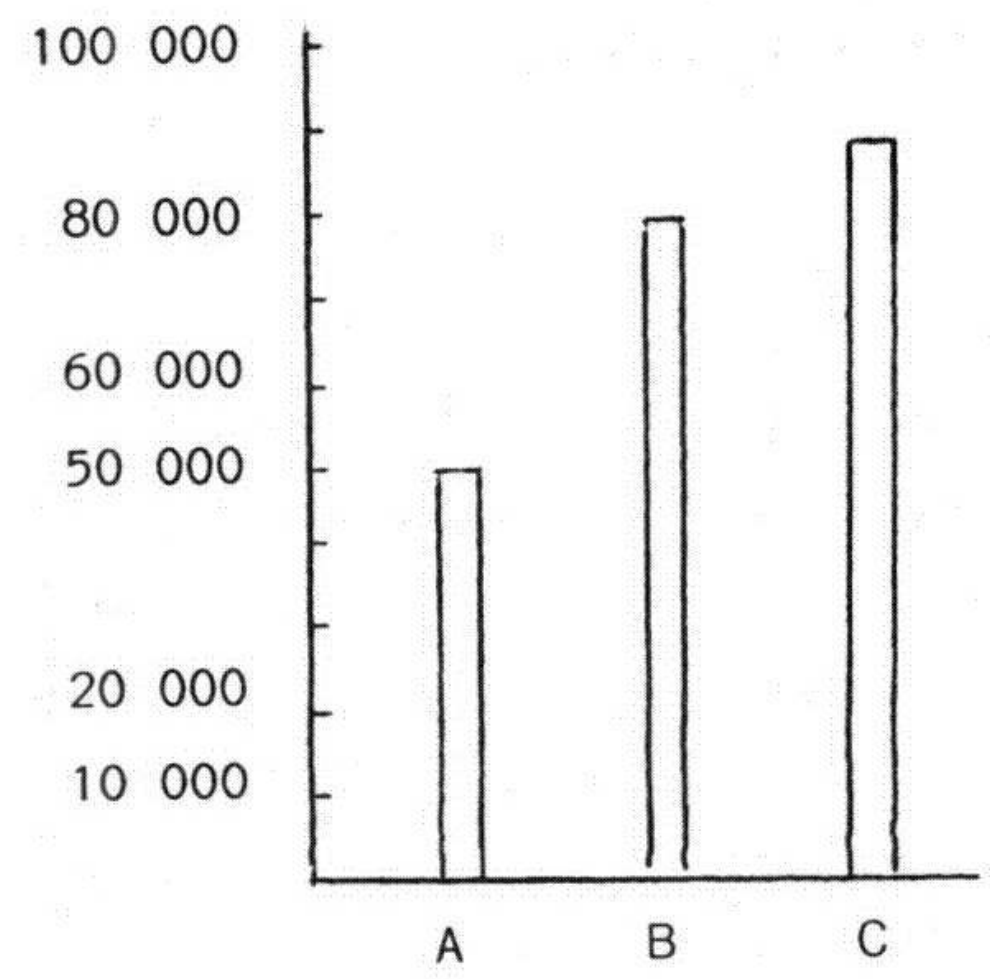

Exemple 2

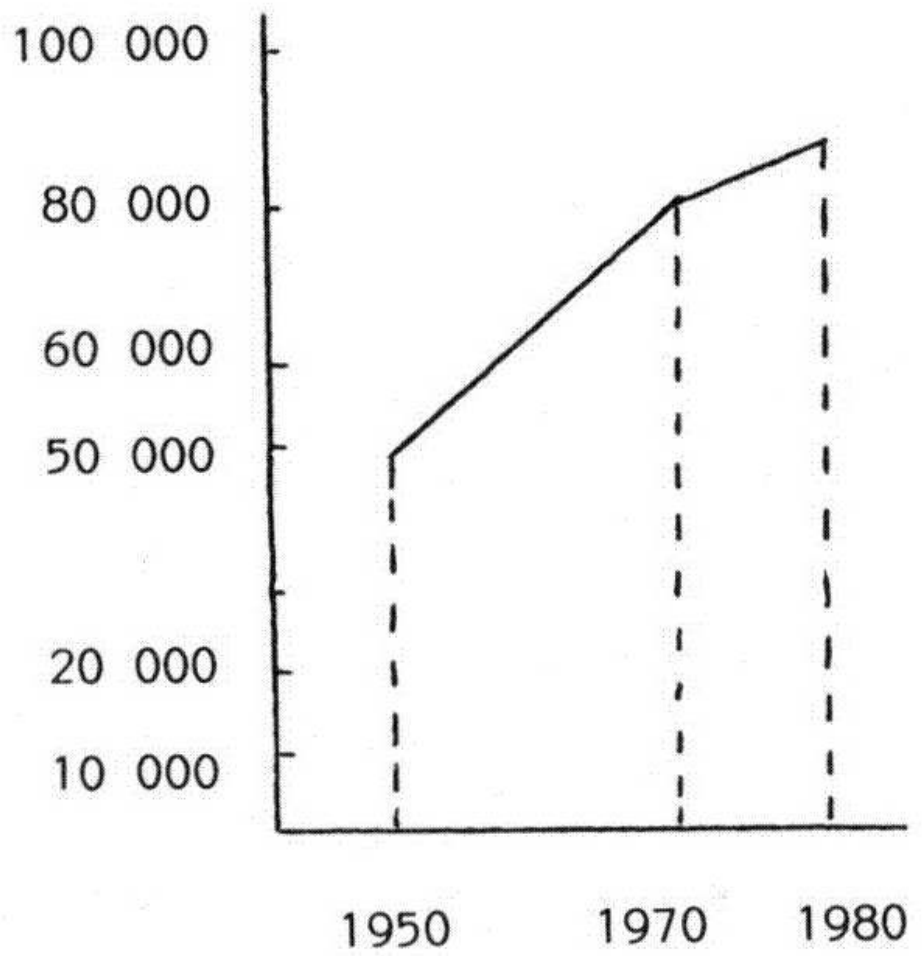

Ainsi, malgré la similitude des nombres cités dans les deux exemples, le graphique 1 (diagramme en bâtons) n'est pas adapté à la situation 2 ; de même le graphique 2 ne 
convient pas pour représenter la situation 1. Cela ne fait pas problème pour les enseignants ; mais la question qui leur est posée (et qui, elle, fait problème) est celle du « comment enseigner cela?». En fait, avant d'espérer apporter une réponse efficace à cette question, une étape est nécessaire : il faut procéder à l'analyse de la tâche soumise aux élèves. Or cette activité, à laquelle les enseignants n'ont pas été formés, est loin d'être triviale dans le cas présent.

En effet, les représentations graphiques constituent un système de signifiants et leur élaboration suppose tout un travail préalable :

- en premier, un travail d'identification de variables ${ }^{5}$;

- puis une tâche d'identification des relations entre ces variables (relations qui sont les « signifiés » du graphique) ;

- enfin, une transcription de ces relations par l'image graphique ${ }^{6}$.

Evidemment, nous ne prétendons pas que cette analyse permette de répondre à la question "comment enseigner les représentations graphiques?». Il nous semble cependant, comme nous le disions plus haut, qu'elle constitue une étape nécessaire dans la définition de l'objet d'enseignement et dans l'élaboration des modalités par lesquelles celui-ci pourrait être efficacement enseigné. Une remarque : actuellement, les professeurs ne sont pas entrainés à ces activités d'analyse de la tâche; on peut donc espérer améliorer ce point précis, par des actions dans le cadre de la formation, initiale ou continue.

\section{Conclusion}

16 Il est relativement banal, aujourd'hui, d'avancer que les relations de l'enseignant au savoir, ses positions épistémologiques (qui sont le plus souvent implicites) jouent un rôle de premier plan dans les choix didactiques qu'il opère et, donc, dans le déroulement même du processus d'enseignement. Le point de vue que nous avons soutenu dans l'exposé s'inscrit en complément de celui-ci : pour nous, les représentations du savoir chez l'enseignant pourraient freiner le développement de l'enseignement de certains secteurs de la connaissance. Le terme de « représentation » est à prendre ici dans un sens très général. En effet, pour chacun des deux domaines sur lesquels nous avons appuyé notre argumentation, il recouvre des aspects différents : en probabilités, l'épistémologie et les conceptions des enseignants seraient en cause ; en revanche, en ce qui concerne les représentations graphiques, les problèmes liés à l'identification du savoir pourraient représenter une source majeure de difficultés.

\section{NOTES}

1. A titre d'exemple, considérons un dé parfait à 6 faces numérotées de 1 à 6 . La probabilité d'apparition d'un nombre pair est estimée par la fréquence relative des sorties de nombres pairs lors d'un grand nombre de jets. 
2. Dans la situation du (1), le nombre de cas favorables est 3 (correspondant aux faces numérotées 2,4 et 6 ), le nombre de cas possibles est 6 . La probabilité est égale à $\mathrm{f}(3 ; 6)$ soit 0,5 .

3. En effet, dans les programmes, on passe fréquemment de l'une à l'autre des deux approches. Dans les programmes de lycées qui se mettent actuellement en place, c'est l'approche empirique qui est retenue.

4. Par enseignement "classique » des probabilités, nous entendons une approche qui combine les aspects empiriques et les aspects théoriques. Elle aspire donc à modéliser certains aspects de la réalité alors que la théorie axiomatique, elle, ignore a priori cette contrainte.

5. Dans le cas de l'exemple 2, les variables sont la date et la population. Il s'agit de grandeurs familières et relativement "simples" pour les élèves de collèges. Mais dans de nombreux exercices scolaires, les difficultés conceptuelles posées par les variables elles-mêmes sont importantes.

6. L'image graphique est généralement transcrite dans un plan lequel, bien souvent, doit être muni d'une structure euclidienne (c'est le cas dans l'exemple 2 si l'on veut que la pente des segments de droite « représente » la croissance de la population).

\section{AUTEUR}

\section{SYLVETTE MAURY}

IREM, université de Montpellier II, USTL 